\title{
Locomotor patterns in cerebellar ataxia
}

2

3 Martino $\mathrm{G}^{1,2}$, Ivanenko $\mathrm{YP}^{2}$, Serrao $\mathrm{M}^{3,4}$, Ranavolo $\mathrm{A}^{5}$, d'Avella $\mathrm{A}^{2}$, Draicchio $\mathrm{F}^{5}$, Conte $\mathrm{C}^{3,6}$, Casali

$4 \quad \mathrm{C}^{4}$, Lacquaniti $\mathrm{F}^{1,2,7}$

5

$6{ }^{1}$ Centre of Space Bio-medicine, University of Rome Tor Vergata, Rome, Italy

$7 \quad{ }^{2}$ Laboratory of Neuromotor Physiology, IRCCS Santa Lucia Foundation, Rome, Italy

$8{ }^{3}$ Rehabilitation Centre Policlinico Italia, Rome, Italy

$9{ }^{4}$ Department of Medical and Surgical Sciences and Biotechnologies, Sapienza University of

10 Rome, Latina, Italy

$11{ }^{5}$ INAIL, Department of Occupational Medicine, Monte Porzio Catone, Rome, Italy

12

${ }^{6}$ IRCCS C. Mondino, Department of Neuroscience, University of Pavia, Pavia, Italy

${ }^{7}$ Department of Systems Medicine, University of Rome Tor Vergata, Rome, Italy

Correspondence to:

24 Dr Giovanni Martino

tel. ++39 06.51.50.14.75

fax. ++3906.51 .50 .14 .82$ 


\section{Abstract}

Several studies demonstrated how cerebellar ataxia (CA) affects gait, resulting in deficits in multi-joint coordination and stability. Nevertheless, how lesions of cerebellum influence the locomotor muscle pattern generation is still unclear. To better understand the effects of CA on locomotor output, here we investigated the idiosyncratic features of the spatiotemporal structure of leg muscle activity and impairments in the biomechanics of CA gait. To this end, we recorded the electromyographic (EMG) activity of 12 unilateral lower limb muscles and analyzed kinematic and kinetic parameters of 19 ataxic patients and 20 age-matched healthy subjects during overground walking. Neuromuscular control of gait in CA was characterized by a considerable widening of EMG bursts and significant temporal shifts in the center of activity due to overall enhanced muscle activation between late swing and mid-stance. Patients also demonstrated significant changes in the intersegmental coordination, an abnormal transient in the vertical ground reaction force and instability of limb loading at heel strike. The observed abnormalities in EMG patterns and foot loading correlated with the severity of pathology (clinical ataxia scale, ICARS) and the changes in the biomechanical output. The findings provide new insights into the physiological role of cerebellum in optimizing the duration of muscle activity bursts and the control of appropriate foot loading during locomotion.

Keywords: Cerebellar ataxia, gait adaptation, muscle activation patterns, central pattern generator, limb loading. 


\section{Introduction}

(1) and Stein, 1977; Grillner et al., 1995; Roberts et al., 1995; Rossignol et al., 1998). Several physiological studies on animals, in fact, have described how lesions at different regions of cerebellum are responsible for different deficits in locomotion (Thach and Bastian, 2004). Medial cerebellar region plays a primary role in static and dynamic balance control and in modulating the rhythmic flexor and extensor muscle activity. The intermediate and lateral cerebellar regions appears to be more important for directing limb placement and fine adjustments to the normal locomotor pattern in novel or complex circumstances or when strong visual guidance is required (Morton and Bastian, 2007).

In humans, the gait impairment characterizing cerebellar ataxia (CA) is often clinically described as "drunken gait", as the clinical features typically observed include a widened base of support and an irregular gait pattern (Holmes, 1939). Several studies investigated the biomechanical characteristics of patients with CA, finding these to consist of decreases in step length, gait speed and ankle torques, increased step width, impaired inter-joint coordination and marked variability of all global and segmental gait parameter values (Palliyath et al., 1998; Mitoma et al., 2000; Earhart and Bastian, 2001; Stolze et al., 2002; Morton and Bastian, 2003; Ilg et al., 2007; Serrao et al., 2012; Wuehr et al., 2013). All these gait abnormalities, reflecting a lack of limb coordination and impaired balance, greatly restrict these patients in their daily life activities and predispose them to falls (Van de Warrenburg et al., 2005b; Nardone and Schieppati, 2010).

No studies have yet been performed to provide a detailed analysis of muscle activity patterns during locomotion in patients with CA. Furthermore, no information on the relationship between the observed kinematic and kinetic abnormalities and the related muscle activity have been provided so far. Therefore, the specific contribution of the cerebellum to the production of locomotor muscle pattern behaviors in humans is still unclear. Impaired processing of sensorimotor information in the cerebellum about foot kinematics and kinetics (Bosco et al., 2006) may also disturb the limb loading and placement. Previous findings suggest that the cerebellum helps in 
modulating sensorimotor interactions, integrates both feedforward and feedback control of balance, and plays a functional role in motor learning and adaptation (Horak and Diener, 1994; Morton and Bastian, 2004; Konczak and Timmann, 2007; Bastian, 2011; Goodworth et al., 2012; Ilg and Timmann, 2013). Thus, lesions of the cerebellum may induce abnormalities in the spatial and temporal pattern of muscle activation resulting in specific gait impairments.

The aim of this study was to provide a wider characterization of ataxic gait by exploring the muscle activation patterns of patients affected by $\mathrm{CA}$, and correlating them with the kinematics, kinetics and the degree of severity of the pathology. The first objective was to test the hypothesis that subjects with cerebellar damage show abnormalities in switching and scaling individual muscles resulting in prolonged activity, as it occurs during upper limb movements in CA (Hallett et al., 1975) or during early development of locomotion (Dominici et al., 2011) when the cerebellum is still immature (Vasudevan et al., 2011). As a secondary objective, we studied the kinematic and kinetic behavior of whole lower limb by analyzing the multiple joint coordination as well as the ground reaction force during loading response. Particularly, we sought to investigate whether the loading response in CA is impaired during weight acceptance, representing the most demanding task to guarantee the initial limb stability and the preservation of progression (Perry, 1992). To assess the coordination deficits, we used the methods developed earlier for normal gait (Borghese et al., 1996; Lacquaniti et al., 2002) and we expected that applying this analysis technique to CA patients may highlight specific alterations in the planar covariation of limb segment elevation angles (Dominici et al., 2010; MacLellan et al., 2011; Leurs et al., 2012).To this end we investigated a sample of patients with primary degenerative pancerebellar diseases. These cerebellar disorders may represent an appropriate model to investigate the role of cerebellum as a whole in locomotor pattern generation. 
Participants

Nineteen patients (5 females and 14 males; age range $32-65 \mathrm{yrs}$, weight $68 \pm 8 \mathrm{~kg}$ [mean \pm $\mathrm{SD}$ ], leg length $0.78 \pm 0.06 \mathrm{~m}$ ) affected by inherited $\mathrm{CA}$, and twenty age-matched healthy subjects HS ( 7 females and 13 males; age range $34-70 \mathrm{yrs}$, weight $70 \pm 14 \mathrm{~kg}$, leg length $0.80 \pm 0.05 \mathrm{~m}$ ) were studied. The characteristics of patients are described in Table 1. Eleven patients had a diagnosis of autosomal dominant ataxia (spinocerebellar ataxia, 7 pts with SCA1, 4 pts with SCA2), while the other 8 had sporadic adult onset ataxia of unknown etiology (SAOA). Even if extracerebellar involvement is common in both SCA1 and 2, none of our patients was found to have clinically significant signs other than cerebellar ones. In particular, they did not show extrapyramidal or pyramidal signs, nor signs of peripheral nerve or muscle deficits, which tend to be overt over the course of the disease. All patients were at a relative early stage of disease as demonstrated by the low International Cooperative Ataxia Rating Scale (ICARS) (Table 1), so that within the limits of clinical ascertainment methods they can be regarded as relatively "pure" cerebellar patients. All patients underwent a complete neurological assessment which included: (i) cognitive evaluation (MMSE, mini-mental state scale); (ii) cranial nerves evaluation; (iii) muscle tone evaluation; (iv) muscle strength evaluation; (iv) joint coordination evaluation; (v) sensory examination; (vi) tendon reflex elicitation; (vii) disease severity measured by ICARS (Trouillas et al., 1997). Particularly, sensation was tested clinically for light touch, pain, joint position and vibration, starting from the toes and moving proximally; touch was tested by a wisp of cotton, pain by a sharp pin, vibration by a $128-\mathrm{Hz}$ tuning fork; proprioception was investigated by asking five times the blinded patient to describe the position of the second toe and the ankle, which were passively moved upward or downward by the examiner, avoiding end-of-range-of-motion position (DeMyer, 2003). In each of these three sensory tests, we assessed whether sensation was normal, reduced or absent, 127 specifying the region of the body. All the patients were evaluated by two experienced neurologists 128 (CC and MS). 
No patient had any kind of visual impairment, in particular no optic atrophy or retinitis pigmentosa were revealed. On the other hand, almost all patients had oculomotor abnormalities such as gaze nystagmus or square waves during pursuit movements, which are common in cerebellar disorders with no obvious impairment of visual acuity. No patient showed clinical features of spasticity, strength deficit, sensory deficit, and/or cognitive impairment (MMSE >26). Furthermore, no relevant inter-limb asymmetries in terms of dysmetria, asynergia and hypotonia and limb kinetic ICARS scores were found between right and left side. All patients showed (at MRI) pancerebellar degenerations with significant atrophy of the cerebellar vermis. Patients enrolled in our study were undergoing physical therapy, which included upper and lower limb exercises, balance and gait training. All participants were capable of walking independently on a level surface, they provided informed written consent prior to taking part in the study, which complied with the Helsinki Declaration and had local ethics committee approval.

\section{Procedures and data recording}

Subjects were asked to walk barefoot along a walkway, approximately $7 \mathrm{~m}$ length, while looking forward. They walked at comfortable self-selected speeds, but were encouraged to walk also at the fastest speed at which they still felt safe, resulting in a range of different speeds across the recorded trials. Given that typical walking speeds were on the slow side in patients, we instructed the healthy control subjects to also walk barefoot at low comfortable speeds $(\sim 30-50 \%$ slower than self-selected speed), in order to roughly match the walking speed in the two groups of subjects. Before the recording session, subjects practiced for a few minutes to familiarize with the procedure. To ensure safe walking conditions, an assistant walked alongside the patients during the trials when necessary. In order to avoid muscle fatigue, groups of three trials were separated by 1 -min rest periods. At least 15 trials were recorded for each subject $(\geq 10$ trials at self-selected speed and $\geq 5$ trials at fast speed in patients or slow speed in controls) and the strides related to gait initiation and termination were discarded so that each trial included from 1 to 3 consecutive gait cycles. Only strides whose speed fell within the range of $3-4.5 \mathrm{~km} / \mathrm{h}$ (since most trials were performed in this range) were retained here for further analysis. Therefore the number of strides 
analyzed in each subject (at matched walking speeds, $3-4.5 \mathrm{~km} / \mathrm{h}$ ) was on average $12.8 \pm 4.6$ for CA patients and $12.7 \pm 3.6$ for control subjects, while the number of excluded strides was on average $18.4 \pm 11.7$ for CA patients and $10.4 \pm 3.8$ for control subjects.

Kinematic data were recorded bilaterally at $300 \mathrm{~Hz}$ using an optoelectronic motion analysis system (SMART-D System, BTS, Milan, Italy) consisting of 8 infrared cameras spaced around the walkway. Twenty-two retro-reflective spherical markers (15 mm in diameter) were attached on anatomical landmarks according to Davis et al. (1991). Anthropometric measurements were taken on each subject. These included the mass and height of the subject and the length of the main segments of the body according to the Winter's method (Winter, 1979). Ground reaction forces were recorded at $1200 \mathrm{~Hz}$ by means of two force platforms $(0.6 \cdot \mathrm{m} \times 0.4 \cdot \mathrm{m}$; Kistler 9286B, Winterthur, Switzerland), placed at the center of the walkway, attached to each other in the longitudinal direction but displaced by $0.2 \cdot \mathrm{m}$ in the lateral direction. The EMG data were recorded at $1000 \mathrm{~Hz}$ using a wireless system (FreeEMG300 System, BTS, Milan, Italy). Bipolar Ag-AgCl surface electrodes were used to record EMG activity from 12 muscles simultaneously on the right side of the body in each subject: tibialis anterior (TA); gastrocnemius lateralis (LG); gastrocnemius medialis (MG); soleus (SOL); peroneus longus (PL); vastus lateralis (VL); vastus medialis (VM); rectus femoris (RF); biceps femoris (BF); semitendinosus (ST); tensor fascia latae (TFL); gluteus medium (GM). Innervation zones and tendon regions were identified using multi-channel highdensity EMG recordings (Barbero et al., 2012) and SENIAM guidelines (Hermens et al., 1999) to ensure correct placement of EMG electrodes. Acquisition of the EMG, kinematic, and kinetic data was synchronized.

\section{Data Analysis}

GAIT CYCLE DEFINITION. Gait cycle was defined as the time between two successive foot contacts of the same leg and foot strike and lift-off events were determined by maximum and minimum excursions of the limb angle (Borghese et al., 1996; Vasudevan et al., 2011), defined as the angle between the vertical axis and the limb segment (from the greater trochanter to lateral malleolus) projected on the sagittal plane. When subjects stepped on the force platforms, these 
kinematic criteria were verified by comparison with foot strike and lift-off measured from a threshold crossing event in the vertical force ( $7 \%$ of body weight). In general, the difference between the time events measured from kinematics and kinetics was no more than $3 \%$. Nevertheless, since the kinematic criterion produced a small error in the identification of stance onset, for averaging and assessing the vertical ground reaction forces when subjects stepped on the force plate, we identified the foot strike from the kinetic data.

KInEMATIC DATA PROCESsing. The following general gait parameters were calculated for each subject: walking speed, cycle duration, relative stance duration, stride length and stride width. The stride length and width were normalized to the limb length (thigh+shank) of each subject. We computed both the anatomical joint angles and the elevation angles of the limb segments relative to the vertical for the right lower limb (Borghese et al., 1996), as well as the pitch and roll angles for the trunk. From these variables, we derived the range of angular motion (RoM). The kinematic data were time interpolated over individual gait cycles to fit a normalized 200-point time base. We also assessed the inter-stride angular variability by calculating the mean standard deviation (SD) of the joint and trunk orientation angles.

Most coordination analyses of gait in CA (Earhart and Bastian, 2001; Stolze et al., 2002; Morton and Bastian, 2003; Ilg et al., 2007) involved the use of angle-angle plots and thus examined movement at two joints at a time. We used a more advanced analysis technique, termed the planar law of intersegmental coordination, which allows to examine movement coordination at the thigh, shank and foot segments simultaneously (Borghese et al., 1996; Lacquaniti et al., 2002). Briefly, the temporal changes of the elevation angles at the thigh, shank, and foot covary during walking. When these angles are plotted in three dimensions (3D), they describe a loop that can be least-squares fitted to a plane over each gait cycle (Borghese et al., 1996). A principal component analysis (PCA) was applied to the group of three segment elevation angle trajectories to determine covariance loop planarity, width and orientation. To this end, we computed the covariance matrix of the ensemble of time-varying elevation angles over each gait cycle. The first two eigenvectors $u_{1}$ and $u_{2}$ lie on the best-fitting plane of angular covariation and the third eigenvector $\left(u_{3}\right)$ is the 
213 normal to the plane and thus defines the plane orientation. The planarity of the trajectories was

214 quantified by the percentage of total variation $\left(P V_{3}\right)$ accounted for by the third eigenvector of the 215 data covariance matrix (for ideal planarity $P V_{3}=0 \%$ ). Covariance loop width was determined using 216 the percent variance $\left(P V_{2}\right)$ explained by the second eigenvector $u_{2}$ since it is oriented in the 217 direction of the minor axis of the loop formed by the elevation angles (if $\mathrm{PV}_{2}$ is small, the thigh218 shank-foot loop tends to be a line, Ivanenko et al., 2008). Covariance plane orientation was 219 quantified using the direction cosine between the third principal axis and the positive semi-axis of 220 the thigh segment $\left(u_{3 t}\right)$, which was found to vary depending on walking conditions (Bianchi et al., 221 1998; Ivanenko et al., 2008) or gait pathology (Grasso et al., 1999, 2004; MacLellan et al., 2011; 222 Leurs et al., 2012). For each subject, the parameters of planar covariation $\left(u_{3 t}, P V_{2}\right.$ and $\left.P V_{3}\right)$ were 223 averaged across strides.

GROUND REACTION FORCES. The steps in which only the right foot stepped onto one of the force plates were analyzed. The vertical ground reaction force (GRF) was calculated and normalized to the body mass (Winter, 1991). In addition, because the lower limb can undergo an impulsive load, the heel strike transient (Verdini et al., 2006) during the weight-acceptance period was evaluated by calculating the peak-to-peak change between the transient maximum and the following local minimum in the GRF $\left(\Delta_{1}\right.$, Fig. 4A). If this transient was absent, the change was considered equal to zero. Since the transient may also be related to limb instability or to small oscillations of the limb during the heel strike, we evaluated the corresponding kinematics correlates: the peak-to-peak change between maximum and minimum values of the vertical velocity of three markers placed on the greater trochanter (hip), lateral femur epicondyle (knee) and lateral malleolus (ankle) during the $0-10 \%$ interval of the stance phase ( $\Delta_{2}$, Fig. 4B). third-order Butterworth filter $(20-450 \mathrm{~Hz})$, rectified and low-pass filtered with a zero-lag fourth-order 
200 points. For each individual, the EMG signal from each muscle was normalized to its peak value across all trials.

To characterize differences in the amplitude and timing of EMG activity between CA and HS groups, we computed the following parameters: mean and maximum EMG activity (in $\mu \mathrm{V}$ ), antagonist co-activation index $(C I)$, center of activity $(C o A)$, and full width at half maximum $(F W H M)$. EMG parameters were calculated over individual strides and then averaged across cycles.

The $C I$ was assessed between the thigh (mean activity of quadriceps RF-VL-VM vs 249 hamstring BF-ST) and calf (mean activity of triceps MG-LG vs TA) antagonistic muscle groups using the following formula (Rudolph et al., 2000; Mari et al., 2014):

$$
C I=\frac{\sum_{\mathrm{j}=1}^{200}\left[\left(\mathrm{EMG}_{\mathrm{H}}(\mathrm{j})+E \mathrm{EMG}_{\mathrm{L}}(\mathrm{j})\right) / 2\right] \times\left(\mathrm{EMG}_{\mathrm{L}}(\mathrm{j}) / \mathrm{EMG}_{\mathrm{H}}(\mathrm{j})\right)}{200}
$$

250

where $E M G_{H}$ and $E M G_{L}$ represent the highest and the lowest activity between the antagonist muscle pairs. In order to have a global measure of the co-activity level, the $C I$ was then averaged over the entire gait cycle $(j=1: 200)$. This method provided a sample-by-sample estimate of the relative activation of the pair of muscles as well as the magnitude of the co-contraction over the entire cycle. Using this equation, high co-contraction values represent a high level of activation of both muscles across a large time interval, whereas low co-contraction values indicate either low level activation of both muscles, or a high level activation of one muscle along with low level activation of the other muscle in the pair (Rudolph et al., 2000).

The $C o A$ during the gait cycle was calculated using circular statistics (Batschelet, 1981) and plotted in polar coordinates (polar direction denoted the phase of the gait cycle, with angle $\theta$ that varies from 0 to $360^{\circ}$ ). The $C o A$ of the EMG waveform was calculated as the angle of the vector (first trigonometric moment) which points to the center of mass of that circular distribution using the following formulas:

$$
A=\sum_{t=1}^{200}\left(\cos \theta_{t} \times E M G_{t}\right)
$$




$$
\begin{aligned}
& B=\sum_{t=1}^{200}\left(\sin \theta_{t} \times E M G_{t}\right) \\
& C o A=\tan ^{-1}(B / A)
\end{aligned}
$$

263 The $C o A$ was chosen because it was impractical to reliably identify a single peak of activity in the 264 majority of muscles, especially in pathological subjects. It can only be considered as a qualitative 265 parameter, because averaging between distinct foci of activity may lead to misleading activity in 266 the intermediate zone. Nevertheless, it can be helpful to understand if the distribution of muscular 267 activity remains unaltered across different groups and muscles.

The FWHM for each EMG waveform was calculated as the sum of the durations of the intervals in which the EMG activity (after subtracting the minimum throughout the gait cycle) exceeded the half of its maximum.

\section{Statistics}

Between groups differences in the spatiotemporal gait parameters, intersegmental coordination, inter-stride variability and $F W H M$ were assessed by performing unpaired two-sample t-tests. The analysis of $\operatorname{CoA}$ was performed using the Watson-Williams test for circular data 276 (Watson and Williams, 1956). The correlation between kinematics, kinetics, muscle activation 277 patterns and clinical scores was performed using Spearman's rank correlation coefficient. The correlation coefficients used in the regression plots were corrected for multiple samples from the same participants. Descriptive statistics included means \pm SD, and significance level was set at $p<0.05$. All statistical analysis were performed using Statistica (v7.0) and custom software written in Matlab (v8.1). 


\section{Results}

General gait parameters and kinematics

At matched walking speeds, cerebellar patients showed a significant increase in the stride width, reduction in the cycle duration and stride length in comparison with healthy controls (Fig. 1A). Instead, the relative stance duration was not significantly different in the two groups. These results are consistent with previous studies (Palliyath et al., 1998; Mitoma et al., 2000; Serrao et al., 2012). Figure 1B shows the ensemble-averaged kinematic patterns. The time course of changes of hip and knee joint angles of CA patients was very similar to that of HS. In contrast, a substantial reduction of the ankle joint excursion $(p<0.00004)$ and consistently larger oscillations in the trunk roll and pitch angles $(p<0.0009)$ were observed in CA relative to HS (Fig. 1C).

Figure $2 \mathrm{~A}$ illustrates the stride-averaged $( \pm S D)$ thigh, shank and foot elevation angles and corresponding gait loops plotted in 3D for one representative HS (left) and one CA patient (right). In both groups, the temporal changes of the elevation angles covary during walking, describing a characteristic loop over each stride that is best-fitted by a plane $\left(P V_{3}<1 \%\right.$, Fig. $\left.2 \mathrm{~B}\right)$. The percentage of variance accounted for by the second eigenvector $\left(P V_{2}\right)$ was significantly greater in CA $(p<0.002)$ indicating a wider gait loop (Fig. 2B). The orientation of the covariance plane $\left(u_{3 t}\right.$ parameter) was also significantly different between the two groups $(p<0.0002)$.

The stride-by-stride variability in gait kinematics was consistently larger in CA. Figure 3A illustrates superimposed plots of the hip, knee, ankle, and trunk pitch and roll angles during individual gait cycles in one control subject and one CA patient. On average, the inter-stride variability in the angles, estimated as the mean SD over the gait cycle, was about $50 \%$ greater in CA patients (Fig. 3B).

\section{Ground reaction forces}

Figure 4A illustrates the vertical component $\left(F_{y}\right)$ of the GRF for both individual strides in single subjects (upper plots) and averaged curves for all subjects (lower plots). Both groups showed the typical two-peaked profile, with a first maximum during the initial stance phase $(\sim 25 \%$ 
of stance phase) and the second one at the end of stance ( $80 \%$ of stance phase). A small

311 additional peak during weight acceptance, at $\sim 10 \%$ of stance, could also be observed (Fig. 4A),

312 consistent with previous studies in healthy subjects (Borghese et al., 1996; Verdini et al., 2006).

313 This peak was prominent in all CA patients, while it was seen only in a few control subjects at

314 these slow to moderate speeds. We quantified the heel strike transient by calculating the $\Delta_{1}$ 315 parameter (Fig. 4A): $\Delta_{1}$ was significantly larger $(p<0.00004)$ in CA compared to HS (Fig. 4C). The 316 horizontal shear forces did not show systematic differences between the two groups and are not 317 reported.

318 We also examined whether the augmented transient in the vertical GRF in CA (Fig. 4A) 319 correlates with kinematic parameters. To this end, we computed the difference between maximum 320 and minimum values of the vertical velocity of the markers located on the hip, knee, and ankle, 321 measured at the beginning of stance $\left(0-10 \%\right.$ of the stance phase, $\Delta_{2}$ in Fig. $\left.4 \mathrm{~B}\right)$. Examples of 322 velocity traces in one control subject and one CA patient are illustrated in Figure 4B. The $\Delta_{2}$ 323 parameter was significantly larger $(p<0.0001)$ for the knee and hip markers in patients (Fig. 4C), 324 consistent with a greater instability of limb loading in CA as shown by the prominent transient in 325 vertical force $\left(\Delta_{1}\right.$, Fig. 4A).

EMG envelopes

We recorded EMG signals from 12 muscles of the right lower limb. Figure 5A illustrates 329 EMG traces in one healthy subject and one CA patient during two consecutive strides. In both 330 groups, EMG activity during walking tended to occur in bursts that were temporally related to 331 specific kinematic/kinetic events: weight acceptance (VM, VL, RF, TFL, GM), limb 332 loading/propulsion (SOL, MG, LG, PL), foot lift (TA), heel strike (ST, BF). The normalized and 333 ensemble-averaged EMGs for the two groups of subjects are illustrated in Figure 5B. We noticed 334 that the EMG profiles in CA patients often differed relative to those in HS in two main features. 335 First, the major bursts tended to be wider (more prolonged) for most muscles. Second, EMG 336 profiles of CA could show some extra bursts, presumably related to gait instability (Fig. 5A). For 337 instance, the activity of hamstring muscles (BF and ST) in patients started earlier (at about $80 \%$ of 
gait cycle) and was prolonged till about $50 \%$ of gait cycle with respect to the healthy subjects (Fig. 5B). A wider activity was also notable in the TA muscle. Similarly, calf muscles (SOL, MG, LG and $\mathrm{PL}$ ) in CA showed activity throughout the whole stance phase, even at the onset of stance, when they are silent in HS.

EMG envelopes in Figure 5B were normalized to the maximum value across all trials, but we also quantified the absolute mean activity and the extent of modulation of activity of leg muscles over the gait cycle. Although large inter-individual variability was observed (in part due to the individual differences in skin impedance), on average the mean and the max amplitude of muscle activity over the gait cycle was about twice greater in CA patients $(p<0.00001$, Fig. 5C), significantly increased activity in CA was found in both proximal and distal muscles.

CA patients showed significantly higher co-activation index values throughout the gait cycle both for RF-VL-VM vs ST-BF $(11.7 \pm 2.8$ for CA and $9.3 \pm 2.1$ for HS; $p<0.01)$ and MG-LG vs TA (15.5 \pm 3.5 for $\mathrm{CA}$ and $8.7 \pm 2.1$ for HS; $p<0.00001$ ) pairs of antagonist muscles (Fig. 5D).

To characterize differences in timing and duration of EMG activity between HS and CA groups, we computed the center of activity $(C o A)$ and full width at half maximum (FWHM) (Fig. 6A, see Methods). The $\operatorname{CoA}$ was similar for many muscles for the two groups of subjects, though it shifted to slightly later phases of the gait cycle (CCW in the polar plots) in proximal muscles (VL, $\mathrm{ST}, \mathrm{BF}$ ), and to earlier phases (CW in the polar plots) in distal muscles (SOL, MG, LG and PL) in CA patients with respect to HS ( $p<0.00001$, Fig. 6C). The analysis of $F W H M$ allowed us to quantify the duration of activity of each muscle. Figure $6 \mathrm{~B}$ shows that most muscles (TA, hamstrings and distal extensors) significantly increased their FWHM in CA patients in comparison with HS. The mean FWHM (across all muscles) in HS and CA was $20 \%$ and $29 \%$ of the gait cycle, respectively. Also, we verified whether the FWHM depended on velocity in the range of analyzed walking speeds $(3-4.5 \mathrm{~km} / \mathrm{h})$. The analysis did not reveal any significant correlation between FWHM (expressed in \% gait cycle) and walking speed in both groups of subjects (Fig. 6C), consistent with scaling of EMG activity with cycle duration (Ivanenko et al., 2004; Cappellini et al., 2006). 
Correlations between EMGs activation patterns, clinical scores and gait parameters in CA

Figure 7A illustrates significant correlations between muscle activation pattern characteristics and kinematic and kinetic parameters in CA patients. We observed significant relationships $(p<0.02)$ between mean FWHM (averaged across all muscles) and cycle duration, stride length, and stride width of CA patients (Fig. 7A). For the intersegmental coordination and angular motion, we found a significant $(p<0.01)$ correlation of $F W H M$ only with $P V_{2}$ (Fig. 7A).

Figure 7B, C and D show the relationship between clinical ICARS measures and gait parameters (which were significantly different between HS and CA). The following parameters correlated significantly with the ICARS score: cycle duration $(p=0.04)$, stride length $(p=0.04)$, stride width $(p=0.03), P V_{2}(p=0.02)$ (Fig. 7B), $\Delta_{1}$ of the GRF $(p=0.02), \Delta_{2}$ of the hip and knee ( $p=0.01$ and $p<0.001$, respectively) (Fig. 7C), and mean FWHM of EMG envelopes $(p=0.002)$ (Fig. 7D). The FWHM was similar among different forms of CA: $29.2 \pm 8.9 \%$ for SCA1, $28.9 \pm 7.1 \%$ 378 for SCA2 and $28.3 \pm 5.7 \%$ for SAOA. While there were significant differences in the interstride 379 variability in the kinematic parameters between CA and HS (Fig. 3), there was no simple 380 relationship between the increased stride-by-stride variability in CA patients and the clinical ICARS 381 measures (see also llg et al., 2007; Serrao et al., 2012). Thus, a large number of gait and muscle 382 pattern parameters that were significantly different between CA and HS gaits (Fig. 1, 2, 4, 6) 383 correlated with the severity of pathology (ICARS score). 


\section{Discussion}

385

386

387

388

389

390

391

392

393

394

395

396

397

398

399

400

401

402

403

404

405

406

407

408

409

410

411

In this study we investigated muscle activity and the biomechanics of locomotion in a group of patients diagnosed with SCA1, SCA2 and SAOA condition of CA. We analyzed the characteristics of EMG activity of 12 unilateral lower limb muscles, correlating them with the clinical score and global and segmental parameters extracted from the kinematics and ground reaction forces. Our findings revealed new idiosyncratic features of the CA gait: significant changes in the intersegmental coordination (Fig. 1C, 2), an abnormal transient in the vertical ground reaction force and instability of limb loading at heel strike (Fig. 4). The marked feature of neuromuscular control of gait in CA was the widening of EMG bursts (Fig. 5, 6). Below we discuss the relationship between the primary deficits and/or compensatory strategies and adaptive changes in the walking behavior and muscle activity patterns.

\section{Kinematics features of CA gait}

Several studies have compared the parameters characterizing locomotion of cerebellar patients with that of healthy controls. The results confirmed high variability of spatiotemporal gait parameters (Fig. 3), wide-base support and reduced cycle duration and stride length (Fig. 1A) that has previously been shown to be distinctive features of ataxic locomotion aimed at compensating the wide oscillations of the center of mass due to poor dynamic balance and stability (Palliyath et al., 1998; Ilg et al., 2007; Serrao et al., 2012; Wuehr et al., 2013). Reduced RoM in the ankle joint in CA could be related to shorter steps (Fig. 1A), impaired intersegmental coordination (wider gait loop, Fig. 2) and/or stiffening of the ankle joint, which would reduce push-off forces, necessary to propel the center of mass forward and upward, and thus destabilize the gait (Morton and Bastian, 2003). Increased trunk sway (Fig. 1C, right panel) can be assumed to be related to multidirectional postural instability (Van de Warrenburg et al., 2005a).

The analysis of the intersegmental coordination in CA revealed significant changes in the covariance plane orientation (expressed by $u_{3 t}$, Fig. 2 right panel) and wider gait loop (expressed by the higher values of $\mathrm{PV}_{2}$, Fig. 2). This is a significant finding, because the orientation of the 
covariance plane reflects a specific tuning of the phase coupling between pairs of limb segments and is related to an ability of the subject to adapt to different walking conditions (Bianchi et al., 1998; Dominici et al., 2010). For instance, in toddlers, the ability to adapt to different terrains is very limited and the maintenance of a roughly constant planar covariance reduces flexibility of the kinematic pattern and thus restricts the manifold of angular segment motion (Dominici et al., 2010). Likewise, it would be of a great interest to study whether there is also a lack of specific adaptation in the planar covariation of limb segments in CA during walking over different terrains (see, for instance, MacLellan et al., 2011).

\section{Foot loading in $\mathrm{CA}$}

In cerebellar patients, the vertical ground reaction force demonstrated a prominent transient following the heel strike (Fig. 4), indicating an abnormal control of limb loading, correlated with the severity of the pathology (Fig. 7C). Even though it can be observed in healthy individuals during fast walking (Borghese et al., 1996), normally the intensity of this impact is adjusted by shockabsorbing reactions at the ankle, knee and hip (Perry, 1992) and it is small or absent at low and normal walking speeds (Fig. 4A, left panels). A similar peak was also found in other pathologies, like osteoarthritis and low back pain (Collins and Whittle, 1989), in amputees (Klodd et al., 2010), and in healthy subjects during unstable walking on a slippery surface (Cappellini et al., 2009).

Despite its deceiving simplicity, human locomotion incorporating an heel strike and appropriate heel-to-toe rolling pattern during stance is a precise and complex motor task that requires learning (Dominici et al., 2007; Ivanenko et al., 2007). The cause of the impaired loading in CA (Fig. 4) may originate from the unbalanced control and preparation to the foot touchdown. An increase in the impact transient could also be a consequence of leg stiffening in CA patients (Mari et al., 2014) or reduced push-off of the contralateral limb in late stance (Serrao et al., 2012). For instance, in amputees, the occurrence of the heel strike transient is evident on the sound side while the prosthetic limb exhibits smooth loading, presumably due to a lack of active push-off from the prosthetic feet in late stance and/or reduced energy storage and return from the prosthetic feet (Klodd et al., 2010). Nevertheless, the weakness of distal extensors does not inevitably result in 
440

441

442

443

444

445

446

447

448

449

450

451

452

453

454

455

456

457

458

459

460

461

462

463

464

465

466

467

the abnormal GRF transient since it was not observed in peripheral neuropathy (Ivanenko et al., 2013a). Further experiments are needed to understand better its biomechanical nature. Whatever the exact biomechanical reasons for the observed phenomenon, the cerebellum may play an important role in the foot loading control. For instance, cats with unilateral section of the dorsal spinocerebellar tract cannot walk on a slippery floor (R.E. Poppele, unpublished observation) as well as cerebellar gait ataxia in humans may result in leg-placement deficit (Morton and Bastian, 2003).

Muscle activation patterns in cerebellar ataxia

Despite that the kinematics and bilateral coordination of leg muscle activity is quite symmetrical in normal healthy subjects, in pathological conditions there might be some differences (Perry, 1992). We did not find any significant difference in the kinematic parameters and clinical assessment on both sides in CA patients. However, although no relevant clinical and kinematic asymmetry were found in our patients we cannot exclude subclinical differences in the EMG patterns between left and right sides. The recordings of unilateral muscle activity, nevertheless, revealed distinctive features of EMG bursts in CA with respect to HS (Fig. 5, 6, 7D).

Although the muscular patterns of CA patients are variable, the analysis of muscle activation patterns showed distinctive features of the CA gait, in particular an increased amplitude (Fig. 5C) and duration (Fig. 6B) of EMG bursts. On average the amplitude of muscle activity over the gait cycle was about twice in CA patients than in healthy subjects (Fig. 5C, see also Mitoma et al., 2000). It is therefore remarkable that significantly larger muscle activation could lead to similar leg movement kinematics and even to smaller angular oscillations in the ankle joint (Fig. 1B, 2A). In addition to a relatively high level of muscle activity in patients (Fig. 5C), the main difference between HS and CA was the duration of the muscle activation periods (Fig. 6B). The enlarged FWHM was observed in all three groups of CA patients (SCA1, SCA2, SAOA). The widening of EMG bursts was somewhat asymmetric since there were also changes in the $\operatorname{CoA}$ (Fig. 6C): the CoA shifted to slightly later phases of the gait cycle in proximal muscles (VL, ST, BF), and to earlier phases in distal muscles (SOL, MG, LG and PL). 

Mari et al., 2014), in part due to EMG widening (Fig. 5), may represent a compensatory strategy useful to provide mechanical stability by stiffening joints. For instance, an abnormal co-contraction pattern has been demonstrated in categories of people who have a great need for active muscular stabilization, such as the elderly (Peterson and Martin, 2010), individuals who have undergone

473 knee arthroplasty (Fallah-Yakhdani et al., 2012), patients with stroke or traumatic brain injury 474 (Chow et al., 2012), and patients with Parkinson's disease (Meunier et al., 2000). Indeed, 475 compared with healthy subjects, ataxic patients needed to activate antagonist muscles more and 476 for a longer period, possibly in an attempt to compensate for instability (Fig. 1C, 3, 4). The 477 observed EMG widening also correlated with a stereotyped biomechanical output of the CA gait 478 (Fig. 7A). However, while leg stiffening might be beneficial in reducing body oscillations during 479 normal posture, in dynamic conditions it may also be detrimental due to a complex nature of 480 balance control during walking. Therefore, an alternative explanation could be that the broader 481 activity bursts are a result of pathology, as suggested by the positive correlation between the 482 severity of pathology (clinical ataxia scale, ICARS) and the FWHM (Fig. 7D). Nevertheless, taking 483 into account the ability of the central nervous system to adapt when faced with a specific gait 484 pathology, often it is difficult to distinguish what primarily comes from pathology and what comes 485 from compensatory mechanisms (Dietz, 2002; Grasso et al., 2004; Ivanenko et al., 2013a). The 486 observed widening of EMG bursts (Fig. 6B) can possibly be compared to other gait disturbances or 487 gait adaptations. For instance, relatively wider EMG bursts are observed in infants (Dominici et al., 488 2011; Ivanenko et al., 2013b), which may be determined at least in part by the developmental state 489 of the cerebellum (Vasudevan et al., 2011). Similarly to ataxic patients, when children start to walk 490 independently their gait is characterized by considerable trunk oscillations, wide swinging arms, 491 high interstride variability and immature foot trajectory characteristics and intersegmental 492 coordination (Ivanenko et al., 2007; Dominici et al., 2010). Maturation of gait is accompanied by a 493 more selective and flexible control of muscles, with shorter activations and an evident separation of 494 the distinct bursts (Dominici et al., 2011; Teulier et al., 2012; Ivanenko et al., 2013b). 
What is the advantage of the 'narrow' bursts in the activation patterns of HS and why are broader bursts adopted in CA patients? Even though the central pattern generation 'timer' produces different relative stance/swing phase durations depending on walking speed (Prochazka and Yakovenko, 2007; Duysens et al., 2013), the duration of the muscle activation patterns is 499 scaled to the duration of the gait cycle (Cappellini et al., 2006). In cerebellar ataxia patients, 500 widening of muscle activation patterns and shifts in the $\operatorname{CoA}$ (Fig. 6) may be a consequence of 501 improper motor planning (feed-forward control) and processing of proprioceptive information 502 (Bastian, 2011) leading to inaccurate movements (Fig. 3) and to the abnormal transient at heel 503 strike (Fig. 4). Broader activation patterns likely imply higher metabolic cost and may also limit 504 adaptation to different walking conditions and coordination with voluntary movements that require 505 appropriate activation timings/duration (Ivanenko et al., 2005).

506 Our findings are consistent with the idea that the cerebellum contributes to optimizing the 507 duration of muscle activation patterns during locomotion. It remains to be determined if the 508 abnormalities discussed here are specific for cerebellar deficit. In this regard, it is worth noting that 509 abnormal prolongation of EMG activity was also observed in the upper limb muscles during elbow

510 flexions in patients with cerebellar deficits and thus may represent a general feature of cerebellar 511 dysmetria (Hallett et al., 1975). Future research can be focused on the mechanisms underlying the 512 observed phenomena for understanding cerebellar physiology and for using these abnormalities as 513 diagnostic tools for the documentation of cerebellar deficits. 
515 Acknowledgements: This work was supported by the Italian Health Ministry, Italian Ministry of 516 University and Research (PRIN project), Italian Space Agency (CRUSOE and COREA grants), and 517 European Union FP7-ICT program (AMARSi grant \#248311). 
Barbero M, Merletti R, Rainoldi A. Atlas of Muscle Innervation Zones: Understanding Surface Electromyography and Its Applications. Springer, 2012.

Bastian AJ. Moving, sensing and learning with cerebellar damage. Curr Opin Neurobiol 21: 596601, 2011.

Batschelet E. Circular Statistics in Biology. Academic Press, 1981.

Bianchi L, Angelini D, Orani GP, Lacquaniti F. Kinematic coordination in human gait: relation to mechanical energy cost. J Neurophysiol 79: 2155-2170, 1998.

Borghese NA, Bianchi L, Lacquaniti F. Kinematic determinants of human locomotion. J Physiol 494: 863-879, 1996.

Bosco G, Eian J, Poppele RE. Phase-specific sensory representations in spinocerebellar activity during stepping: evidence for a hybrid kinematic/kinetic framework. Exp Brain Res 175: 83-96, 2006.

Cappellini G, Ivanenko YP, Dominici N, Poppele RE, Lacquaniti F. Motor Patterns During Walking on a Slippery Walkway. J Neurophysiol 103: 746-760, 2009.

Cappellini G, Ivanenko YP, Poppele RE, Lacquaniti F. Motor patterns in human walking and running. J Neurophysiol 95: 3426-3437, 2006.

Chow JW, Yablon SA, Stokic DS. Coactivation of ankle muscles during stance phase of gait in patients with lower limb hypertonia after acquired brain injury. Clin Neurophysiol Off J Int Fed Clin Neurophysiol 123: 1599-1605, 2012.

Collins JJ, Whittle MW. Impulsive forces during walking and their clinical implications. Clin Biomech Bristol Avon 4: 179-187, 1989.

Davis III RB, Õunpuu S, Tyburski D, Gage JR. A gait analysis data collection and reduction technique. Hum Mov Sci 10: 575-587, 1991.

DeMyer W. Technique of the Neurologic Examination, Fifth Edition. McGraw Hill Professional, 2003.

Dietz V. Proprioception and locomotor disorders. Nat Rev Neurosci 3: 781-790, 2002.

Dominici N, Ivanenko YP, Cappellini G, d' Avella A, Mondì V, Cicchese M, Fabiano A, Silei T, Di Paolo A, Giannini C, Poppele RE, Lacquaniti F. Locomotor primitives in newborn babies and their development. Science 334: 997-999, 2011.

Dominici N, Ivanenko YP, Cappellini G, Zampagni ML, Lacquaniti F. Kinematic strategies in newly walking toddlers stepping over different support surfaces. J Neurophysiol 103: 1673-1684, 2010.

Dominici N, Ivanenko YP, Lacquaniti F. Control of foot trajectory in walking toddlers: adaptation to load changes. J Neurophysiol 97: 2790-2801, 2007.

Duysens J, De Groote F, Jonkers I. The flexion synergy, mother of all synergies and father of new models of gait. Front Comput Neurosci 7: 14, 2013. 
Earhart GM, Bastian AJ. Selection and coordination of human locomotor forms following cerebellar damage. J Neurophysiol 85: 759-769, 2001.

Fallah-Yakhdani HR, Abbasi-Bafghi H, Meijer OG, Bruijn SM, van den Dikkenberg N, Benedetti M-G, van Dieën JH. Determinants of co-contraction during walking before and after arthroplasty for knee osteoarthritis. Clin Biomech Bristol Avon 27: 485-494, 2012.

Goodworth AD, Paquette C, Jones GM, Block EW, Fletcher WA, Hu B, Horak FB. Linear and angular control of circular walking in healthy older adults and subjects with cerebellar ataxia. Exp Brain Res 219: 151-161, 2012.

Grasso R, Ivanenko YP, Zago M, Molinari M, Scivoletto G, Castellano V, Macellari V, Lacquaniti F. Distributed plasticity of locomotor pattern generators in spinal cord injured patients. Brain J Neurol 127: 1019-1034, 2004.

Grasso R, Peppe A, Stratta F, Angelini D, Zago M, Stanzione P, Lacquaniti F. Basal ganglia and gait control: apomorphine administration and internal pallidum stimulation in Parkinson's disease. Exp Brain Res 126: 139-148, 1999.

Grillner S, Deliagina T, Ekeberg O, el Manira A, Hill RH, Lansner A, Orlovsky GN, Wallén P. Neural networks that co-ordinate locomotion and body orientation in lamprey. Trends Neurosci 18: 270-279, 1995.

Hallett M, Shahani BT, Young RR. EMG analysis of patients with cerebellar deficits. J Neurol Neurosurg Psychiatry 38: 1163-1169, 1975.

Hermens HJ, Freriks B, Merletti R, Stegeman D, Blok J, Rau G, Disselhorst-Klug C, Hägg G. European recommendations for surface electromyography [Online]. Roessingh Research and Development The Netherlands. http://www.seniam.org/pdf/contents8.PDF [6 Feb. 2014].

Holmes G. The Cerebellum of Man. Brain 62: 1-30, 1939.

Horak FB, Diener HC. Cerebellar control of postural scaling and central set in stance. $J$ Neurophysiol 72: 479-493, 1994.

Ilg W, Golla H, Thier P, Giese MA. Specific influences of cerebellar dysfunctions on gait. Brain J Neurol 130: 786-798, 2007.

Ilg W, Timmann D. Gait ataxia-specific cerebellar influences and their rehabilitation. Mov Disord 28: 1566-1575, 2013.

Ivanenko YP, d' Avella A, Poppele RE, Lacquaniti F. On the origin of planar covariation of elevation angles during human locomotion. J Neurophysiol 99: 1890-1898, 2008.

Ivanenko YP, Cappellini G, Dominici N, Poppele RE, Lacquaniti F. Coordination of locomotion with voluntary movements in humans. J Neurosci Off J Soc Neurosci 25: 7238-7253, 2005.

Ivanenko YP, Cappellini G, Solopova IA, Grishin AA, MacLellan MJ, Poppele RE, Lacquaniti F. Plasticity and modular control of locomotor patterns in neurological disorders with motor deficits. Front Comput Neurosci 7, 2013a.

Ivanenko YP, Dominici N, Cappellini G, Paolo AD, Giannini C, Poppele RE, Lacquaniti F. Changes in the Spinal Segmental Motor Output for Stepping during Development from Infant to Adult. J Neurosci 33: 3025-3036, 2013b.

Ivanenko YP, Dominici N, Lacquaniti F. Development of independent walking in toddlers. Exerc Sport Sci Rev 35: 67-73, 2007. 
Ivanenko YP, Poppele RE, Lacquaniti F. Five basic muscle activation patterns account for muscle activity during human locomotion. J Physiol 556: 267-282, 2004.

Klodd E, Hansen A, Fatone S, Edwards M. Effects of prosthetic foot forefoot flexibility on gait of unilateral transtibial prosthesis users. J Rehabil Res Dev 47: 899-910, 2010.

Konczak J, Timmann D. The effect of damage to the cerebellum on sensorimotor and cognitive function in children and adolescents. Neurosci Biobehav Rev 31: 1101-1113, 2007.

Lacquaniti F, Ivanenko YP, Zago M. Kinematic control of walking. Arch Ital Biol 140: 263-272, 2002.

Lennard PR, Stein PS. Swimming movements elicited by electrical stimulation of turtle spinal cord. I. Low-spinal and intact preparations. J Neurophysiol 40: 768-778, 1977.

Leurs F, Bengoetxea A, Cebolla AM, De Saedeleer C, Dan B, Cheron G. Planar covariation of elevation angles in prosthetic gait. Gait Posture 35: 647-652, 2012.

MacLellan MJ, Dupré N, McFadyen BJ. Increased obstacle clearance in people with ARCA-1 results in part from voluntary coordination changes between the thigh and shank segments. Cerebellum Lond Engl 10: 732-744, 2011.

Mari S, Serrao M, Casali C, Conte C, Martino G, Ranavolo A, Coppola G, Draicchio F, Padua L, Sandrini G, Pierelli F. Lower limb antagonist muscle co-activation and its relationship with gait parameters in cerebellar ataxia. Cerebellum Lond Engl 13: 226-236, 2014.

Meunier S, Pol S, Houeto JL, Vidailhet M. Abnormal reciprocal inhibition between antagonist muscles in Parkinson's disease. Brain J Neurol 123 ( Pt 5): 1017-1026, 2000.

Mitoma H, Hayashi R, Yanagisawa N, Tsukagoshi H. Characteristics of parkinsonian and ataxic gaits: a study using surface electromyograms, angular displacements and floor reaction forces. $J$ Neurol Sci 174: 22-39, 2000.

Morton SM, Bastian AJ. Relative Contributions of Balance and Voluntary Leg-Coordination Deficits to Cerebellar Gait Ataxia. J Neurophysiol 89: 1844-1856, 2003.

Morton SM, Bastian AJ. Cerebellar control of balance and locomotion. Neurosci Rev J Bringing Neurobiol Neurol Psychiatry 10: 247-259, 2004.

Morton SM, Bastian AJ. Mechanisms of cerebellar gait ataxia. Cerebellum Lond Engl 6: 79-86, 2007.

Nardone A, Schieppati M. The role of instrumental assessment of balance in clinical decision making. Eur J Phys Rehabil Med 46: 221-237, 2010.

Palliyath S, Hallett M, Thomas SL, Lebiedowska MK. Gait in patients with cerebellar ataxia. Mov Disord Off J Mov Disord Soc 13: 958-964, 1998.

Perry J. Gait analysis: normal and pathological function. Thorofare, NJ: SLACK, 1992.

Peterson DS, Martin PE. Effects of age and walking speed on coactivation and cost of walking in healthy adults. Gait Posture 31: 355-359, 2010.

Prochazka A, Yakovenko S. The neuromechanical tuning hypothesis. Prog Brain Res 165: 255265, 2007. 
Roberts A, Tunstall MJ, Wolf E. Properties of networks controlling locomotion and significance of voltage dependency of NMDA channels: stimulation study of rhythm generation sustained by positive feedback. J Neurophysiol 73: 485-495, 1995.

Rossignol S, Chau C, Brustein E, Giroux N, Bouyer L, Barbeau H, Reader TA.

Pharmacological activation and modulation of the central pattern generator for locomotion in the cat. Ann N Y Acad Sci 860: 346-359, 1998.

Rudolph KS, Axe MJ, Snyder-Mackler L. Dynamic stability after ACL injury: who can hop? Knee Surg Sports Traumatol Arthrosc Off J ESSKA 8: 262-269, 2000.

Serrao M, Pierelli F, Ranavolo A, Draicchio F, Conte C, Don R, Di Fabio R, LeRose M, Padua L, Sandrini G, Casali C. Gait pattern in inherited cerebellar ataxias. Cerebellum Lond Engl 11: 194-211, 2012.

Stolze H, Klebe S, Petersen G, Raethjen J, Wenzelburger R, Witt K, Deuschl G. Typical features of cerebellar ataxic gait. J Neurol Neurosurg Psychiatry 73: 310-312, 2002.

Teulier C, Sansom JK, Muraszko K, Ulrich BD. Longitudinal changes in muscle activity during infants' treadmill stepping. J Neurophysiol 108: 853-862, 2012.

Thach WT, Bastian AJ. Role of the cerebellum in the control and adaptation of gait in health and disease. Prog Brain Res 143: 353-366, 2004.

Trouillas P, Takayanagi T, Hallett M, Currier RD, Subramony SH, Wessel K, Bryer A, Diener HC, Massaquoi S, Gomez CM, Coutinho P, Ben Hamida M, Campanella G, Filla A, Schut L, Timann D, Honnorat J, Nighoghossian N, Manyam B. International Cooperative Ataxia Rating Scale for pharmacological assessment of the cerebellar syndrome. The Ataxia

Neuropharmacology Committee of the World Federation of Neurology. J Neurol Sci 145: 205-211, 1997.

Vasudevan EVL, Torres-Oviedo G, Morton SM, Yang JF, Bastian AJ. Younger Is Not Always Better: Development of Locomotor Adaptation from Childhood to Adulthood. J Neurosci 31: 30553065, 2011.

Verdini F, Marcucci M, Benedetti MG, Leo T. Identification and characterisation of heel strike transient. Gait Posture 24: 77-84, 2006.

Van de Warrenburg BPC, Bakker M, Kremer BPH, Bloem BR, Allum JHJ. Trunk sway in patients with spinocerebellar ataxia. Mov Disord Off J Mov Disord Soc 20: 1006-1013, 2005a.

Van de Warrenburg BPC, Steijns JAG, Munneke M, Kremer BPH, Bloem BR. Falls in degenerative cerebellar ataxias. Mov Disord Off J Mov Disord Soc 20: 497-500, 2005b.

Watson GS, Williams EJ. On the Construction of Significance Tests on the Circle and the Sphere. Biometrika 43: 344, 1956.

Winter DA. Biomechanics of human movement. Wiley, 1979.

Winter DA. The biomechanics and motor control of human gait: normal, elderly and pathological. University of Waterloo Press, 1991.

Wuehr M, Schniepp R, Ilmberger J, Brandt T, Jahn K. Speed-dependent temporospatial gait variability and long-range correlations in cerebellar ataxia. Gait Posture 37: 214-218, 2013. 
677 Figure 1. Kinematics gait parameters. A: comparison of general gait parameters for healthy 678 subjects (HS) and cerebellar ataxic patients (CA). B: ensemble-averaged (mean \pm SD) hip, knee 679 and ankle joint angles and trunk roll and pitch orientation angles in 19 ataxic patients (right) and 20 680 age-matched healthy subjects (left). Data were normalized to the cycle duration and represented in percent of gait cycle. C: range of angular motion (RoM). Asterisks denote significant group differences ( $p<0.05$, un-paired t-tests).

683

Figure 2. Planar covariation of elevation angles during walking in HS and CA. A: ensemble685 averaged (across strides) thigh, shank and foot elevation angles (mean \pm SD) plotted vs. normalized gait cycle and corresponding 3D gait loops and interpolation planes in one healthy subject (left) and one ataxic patient (right). Gait loops are obtained by plotting the thigh waveform vs. the shank and foot waveforms (after mean values subtraction). Gait cycle paths progress in time in the counterclockwise direction, heel touch-down and toe-off phases corresponding roughly to the top and bottom of the loops, respectively. The interpolation planes result from orthogonal planar regression. B: percent of total variation explained by second and third principal component 692 ( $P V_{2}$ and $P V_{3}$, respectively) and $u_{3 t}$ parameter that characterizes the orientation of the normal to 693 the plane are indicated for each group of subjects (mean + SD). Asterisks represent significant group difference $(p<0.05)$.

695

Figure 3. Inter-stride angular variability. A: examples of joint and trunk orientation angles in CA patient (p14) and an age-matched control (h1). Every trace refers to a single cycle. B: inter-stride variability of joint and trunk orientation angles (meanSD: estimated as the mean SD of angular waveforms across strides averaged over all time points of the gait cycle) in healthy subjects and ataxic patients (mean $+S D)$. Asterisks represent significant group difference $(p<0.05)$. Note higher inter-stride variability of angular motion in CA. 
Figure 4. Vertical ground reaction forces (GRF) during walking. A: upper panels: vertical GRF in

704

705

706

707

708

709

710

711

712

713

714

715

716

717

718

719

720

721

722

723

724

725

726

727

728

729

730

one representative healthy subject (top left) and one ataxic patient (top right). Every trace refers to a single cycle. Bottom panels: Ensemble-averaged (mean $\pm \mathrm{SD}$ ) vertical ground reaction forces in healthy subjects $(n=20)$ and ataxic patients $(n=19)$. The patterns are normalized to body weight and plotted vs. normalized stance. Note the prominent transient $\left(\Delta_{1}\right)$ during the weight-acceptance period (marked by a shaded area) in ataxic patients. B: vertical velocity of the markers at hip, knee and ankle joints in one healthy subject (left) and one representative ataxic patient (right) over the time interval around the foot-strike event (from $5 \%$ of stance prior to the heel contact to $15 \%$ after the heel contact). Each trace refers to a single step. $\Delta_{2}$ - peak-to-peak amplitude of velocity traces over first $10 \%$ of stance duration. C: peak-to-peak amplitude (mean + SD) of force transient $\left(\Delta_{1}\right)$ and velocity traces $\left(\Delta_{2}\right)$ during the initial weight acceptance phase of stance in healthy subjects and ataxic patients. Asterisks denote significant group differences $(p<0.05)$.

Figure 5. EMG activity in HS and CA. A: examples of EMG traces in one healthy subject (h4, $4.1 \mathrm{~km} / \mathrm{h})$ and one CA patient $(\mathrm{p} 15,3.4 \mathrm{~km} / \mathrm{h})$ during two consecutive strides. The stance phase is evidenced by a shaded region. B: ensemble-averaged (mean \pm SD) EMG activity patterns of 12 ipsilateral leg muscles recorded from healthy subjects and ataxic patients. EMGs were normalized to their max value across all trials. C: max and mean EMG levels (mean $+\mathrm{SD}$ ) in microvolts. Note higher level of activity in CA. D: co-activation indexes (CI) of "RF-VL-VM vs ST-BF" and "MG-LG vs TA" pairs of antagonist muscles. Asterisks denote significant group differences $(p<0.05)$.

Figure 6. Characteristics of EMG activity. A: schematic description of the evaluation method in one representative EMG envelope of the biceps femoris muscle plotted in the polar coordinates. Full width at half maximum $(F W H M)$ was calculated as the duration of the interval (in percent of gait cycle) in which EMG activity exceeded half of its maximum. In the few cases in which two bursts of activity were present (e.g. in TA), FWHM was calculated as a sum of the durations of the intervals in which EMG activity exceeded half of its maximum. The center of activity $(C o A)$ vector was calculated as the first trigonometric moment of the circular distribution (Batschelet, 1981). B: 
731 FWHM (mean + SD) of 12 EMGs in HS and CA. C: correlation between FWHM and walking 732 speed, the data for all subjects and all individual strides were pooled together (each point 733 represents the individual stride value). Linear regression lines with corresponding $r$ and $p$ values 734 are reported. D: CoA of leg muscle EMGs in healthy subjects (green) and ataxic patients (red). 735 Polar direction denotes the relative time over the gait cycle (time progresses clockwise), the width 736 of the sector represents angular SD across subjects while the radius of the sector indicates the 737 mean angular SD across strides (the smaller the radius the larger the interstride variability). 738 Asterisks denote significant differences between the groups.

739

740 Figure 7. Correlations between gait parameters, EMG burst widening and clinical scores. Only 741 parameters that differed significantly between $\mathrm{HS}$ and CA individuals are plotted in this figure. 742 Each point represents the stride-averaged value for the individual patient. Linear regression lines 743 with corresponding $r$ and $p$ values are reported. A: Relationships between cycle duration, stride 744 length, stride width and $\mathrm{PV}_{2}$ parameter of the intersegmental coordination and mean $F W H M$ of 745 muscle activation patterns (mean FWHM was calculated as the mean across FWHM of all 12 746 muscles). B: gait kinematic parameters vs. the ICARS score. C: parameters of the transient 747 following the heel strike (GRF $\Delta_{1}$, hip $\Delta_{2}$ and knee $\Delta_{2}$, Fig. 4). D: averaged $F W H M$ across all 748 EMGs vs. the ICARS score. 
Table 1. Patients' characteristics. Cerebellar patients were rated using the ICARS score (Trouillas

750 et al., 1997). The table lists the total ICARS scores and the subscores for posture, gait and limb

751 kinetics (we summed up the gait and posture scores to obtain an indicator of balance deficit).

752 Higher scores indicate more severe ataxia.

\begin{tabular}{|c|c|c|c|c|c|c|c|c|c|c|}
\hline \multirow{2}{*}{ Patients } & \multirow{2}{*}{ Age (yr) } & \multirow{2}{*}{ Gender } & \multirow{2}{*}{ BW (kg) } & \multirow{2}{*}{ Diagnosis } & \multirow{2}{*}{$\begin{array}{c}\text { Age at } \\
\text { onset (yr) }\end{array}$} & \multicolumn{5}{|c|}{ ICARS } \\
\hline & & & & & & Gait & Posture & Balance & Lower Limb & Total \\
\hline $\mathrm{P} 1$ & 42 & M & 65 & SCA1 & 35 & 1 & 1 & 2 & 1 & 6 \\
\hline $\mathrm{P} 2$ & 41 & M & 64 & SCA1 & 33 & 1 & 1 & 2 & 1 & 6 \\
\hline P3 & 48 & M & 74 & SAOA & 30 & 1 & 0 & 1 & 1 & 6 \\
\hline P4 & 32 & M & 50 & SCA1 & 28 & 2 & 4 & 6 & 0 & 7 \\
\hline P5 & 33 & M & 52 & SCA1 & 30 & 3 & 3 & 6 & 0 & 7 \\
\hline P6 & 57 & $M$ & 65 & SAOA & 47 & 3 & 4 & 7 & 4 & 12 \\
\hline P7 & 65 & $\mathrm{~F}$ & 65 & SAOA & 62 & 3 & 5 & 8 & 0 & 12 \\
\hline P8 & 59 & $\mathrm{~F}$ & 61 & SAOA & 55 & 3 & 4 & 7 & 1 & 12 \\
\hline P9 & 43 & $\mathrm{~F}$ & 66 & SCA2 & 37 & 5 & 5 & 10 & 2 & 17 \\
\hline P10 & 49 & M & 73 & SCA1 & 41 & 4 & 5 & 9 & 2 & 18 \\
\hline P11 & 46 & M & 77 & SAOA & 17 & 4 & 5 & 9 & 2 & 18 \\
\hline P12 & 37 & M & 67 & SCA2 & 30 & 3 & 6 & 9 & 2 & 20 \\
\hline P13 & 45 & M & 79 & SCA1 & 27 & 3 & 3 & 6 & 3 & 21 \\
\hline P14 & 45 & M & 70 & SCA2 & 35 & 4 & 8 & 12 & 5 & 21 \\
\hline P15 & 44 & $\mathrm{M}$ & 68 & SCA2 & 33 & 5 & 7 & 12 & 5 & 21 \\
\hline P16 & 52 & M & 85 & SCA1 & 40 & 4 & 5 & 8 & 4 & 23 \\
\hline P17 & 45 & $M$ & 68 & SAOA & 30 & 4 & 9 & 13 & 7 & 26 \\
\hline P18 & 62 & $\mathrm{~F}$ & 70 & SAOA & 50 & 5 & 9 & 14 & 7 & 28 \\
\hline P19 & 54 & $\mathrm{~F}$ & 66 & SAOA & 40 & 5 & 10 & 15 & 8 & 30 \\
\hline
\end{tabular}


A

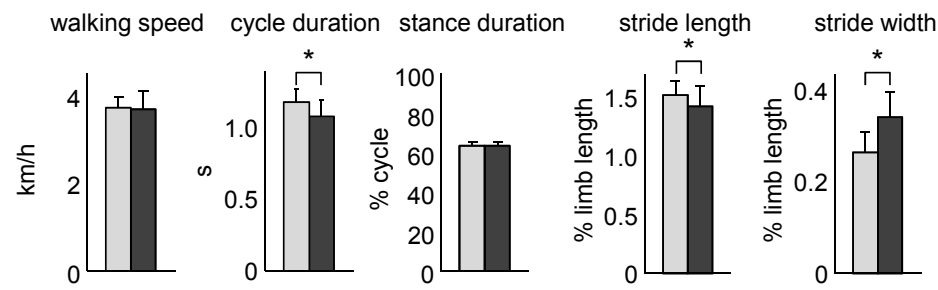

B

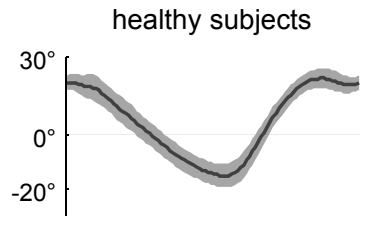

CA patients
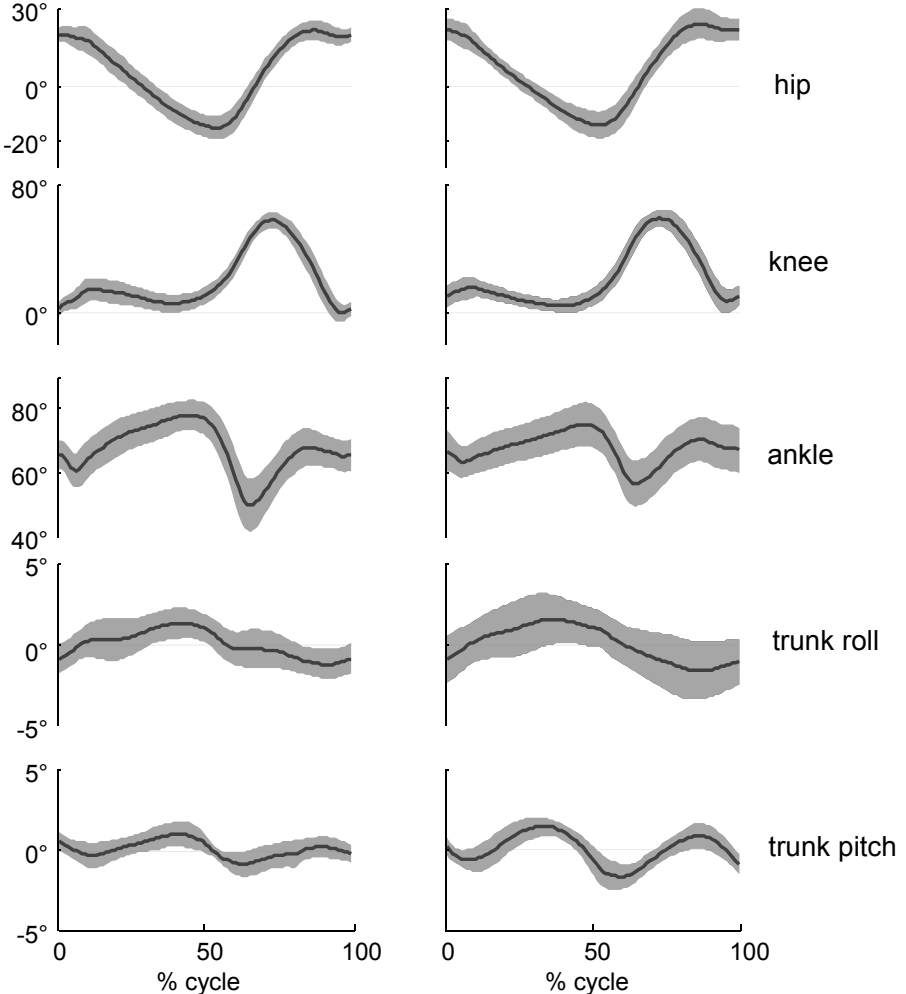

C

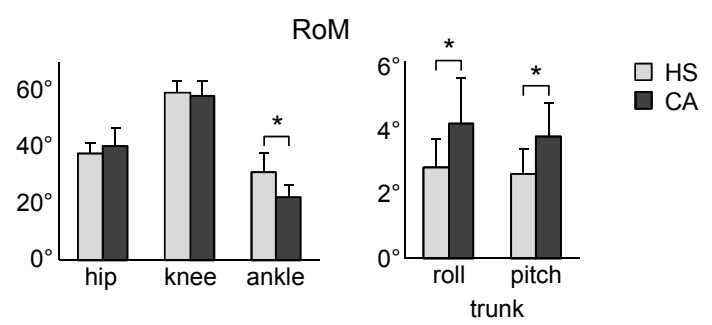

Fig. 1 

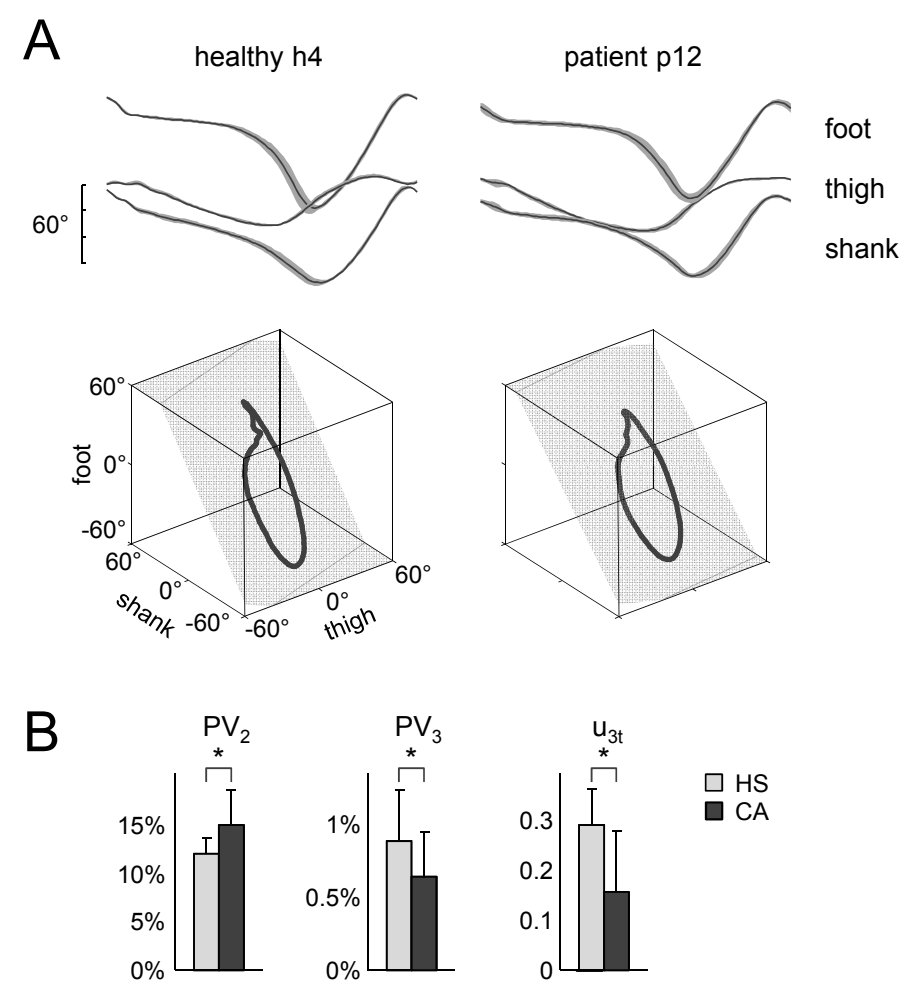

Fig. 2 
A
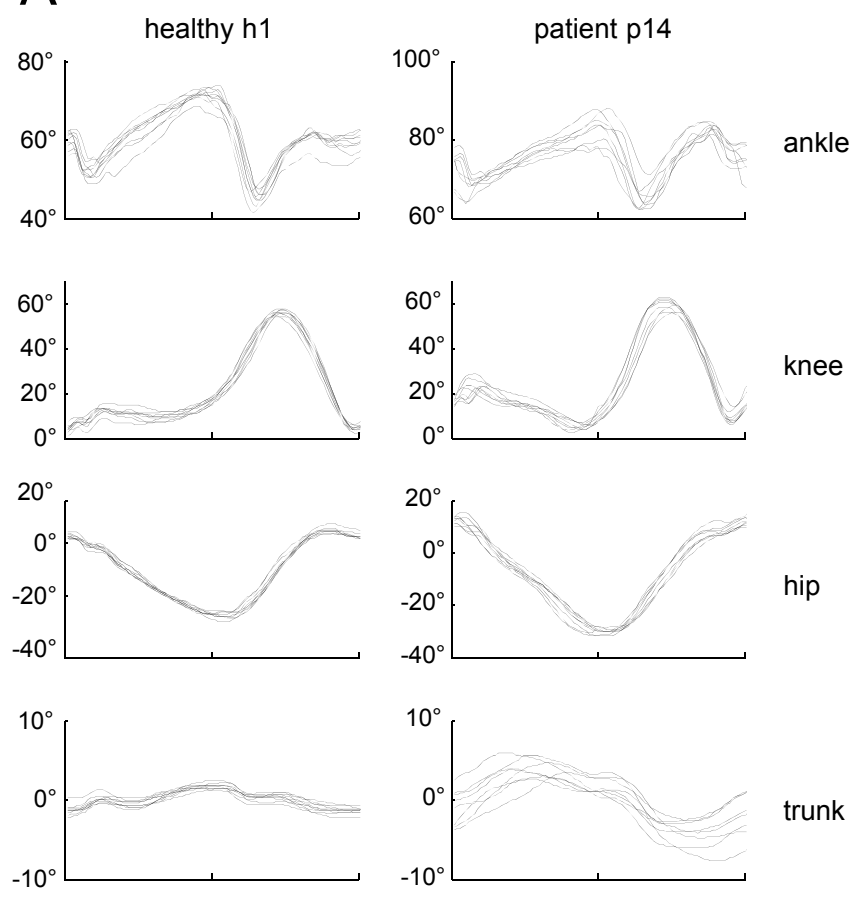

trunk roll
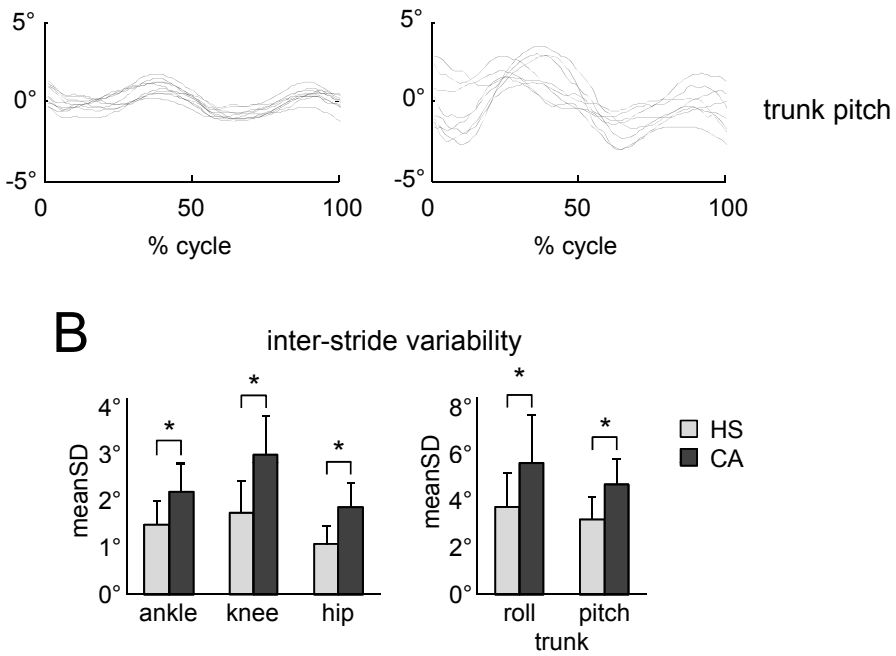

Fig. 3 


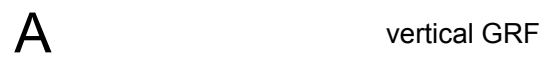

B vertical velocity
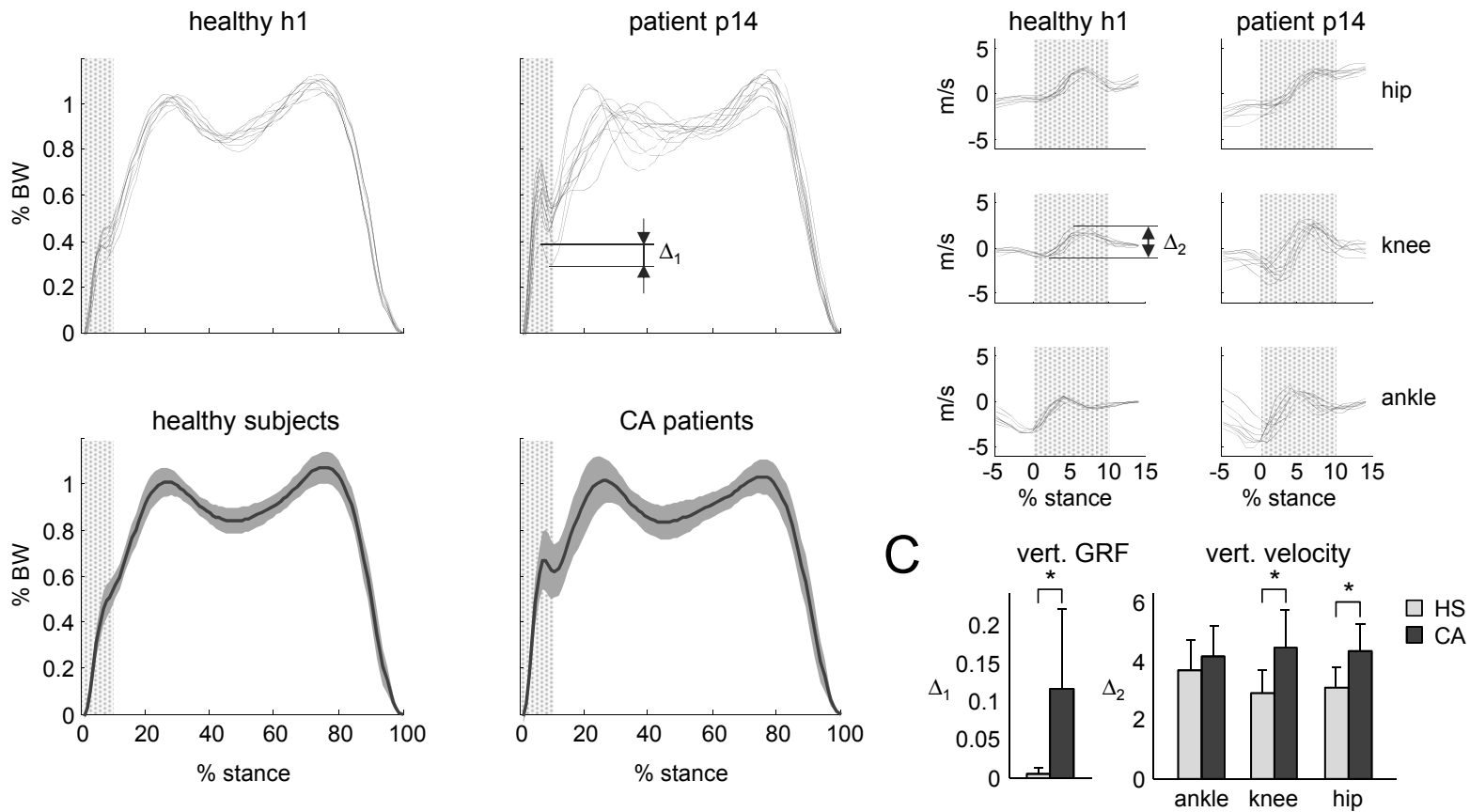

Fig. 4 

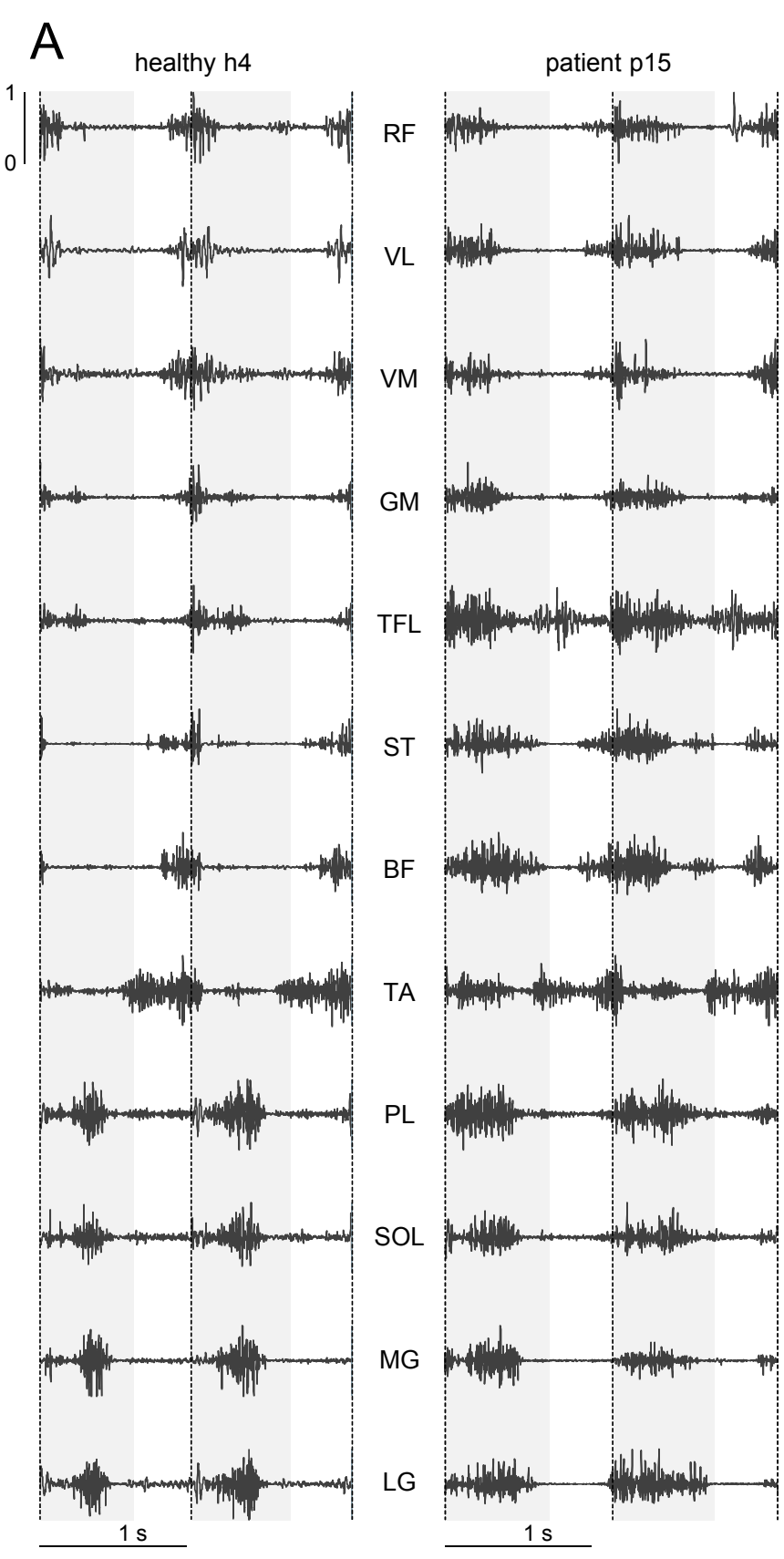

C

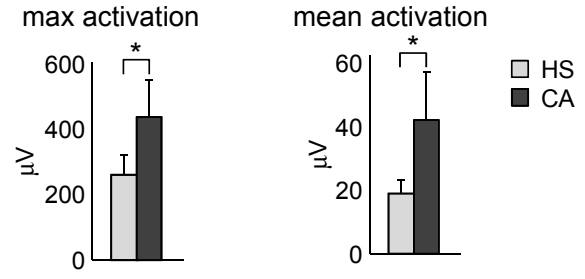

B
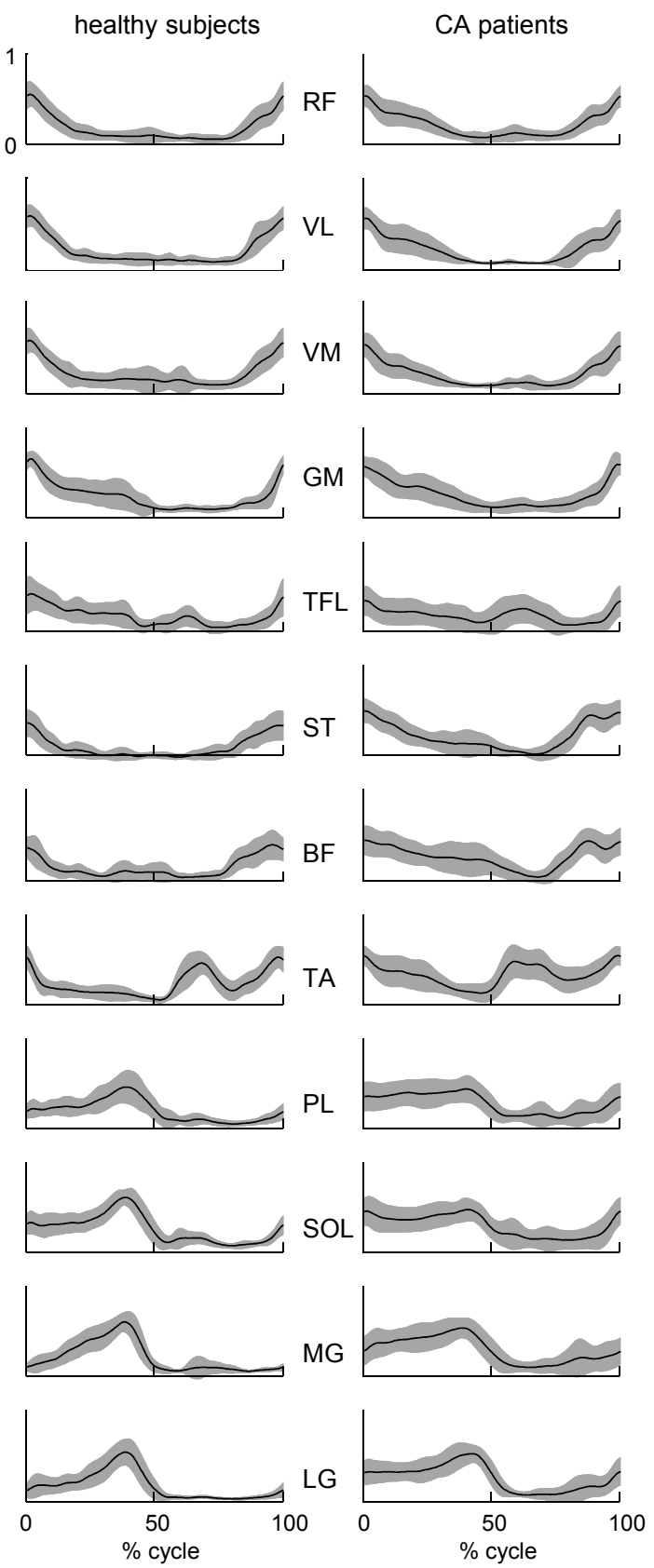

D co-activation index (Cl)

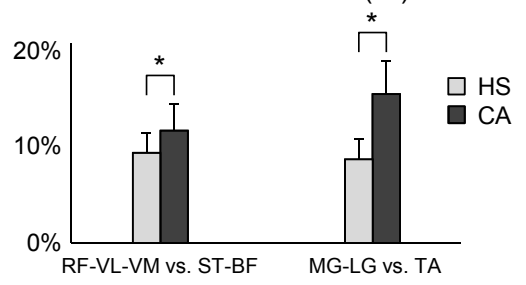

Fig. 5 

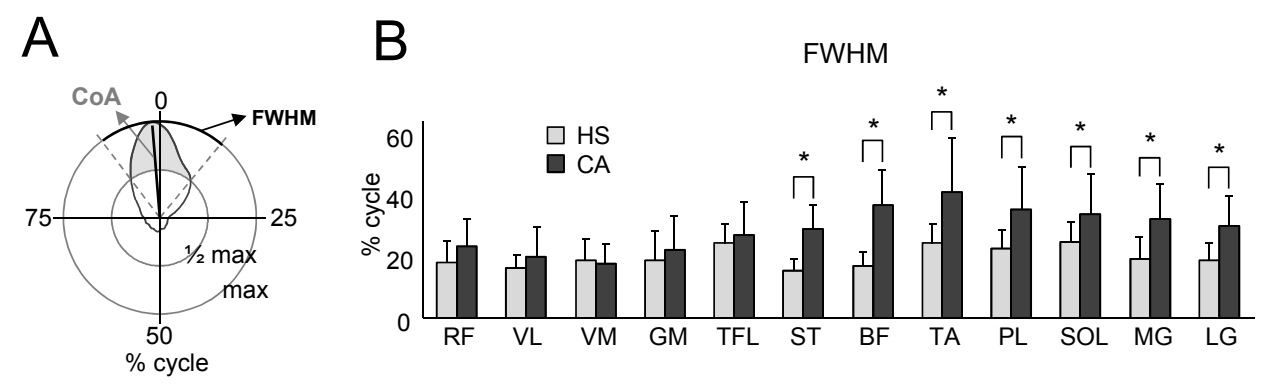

C

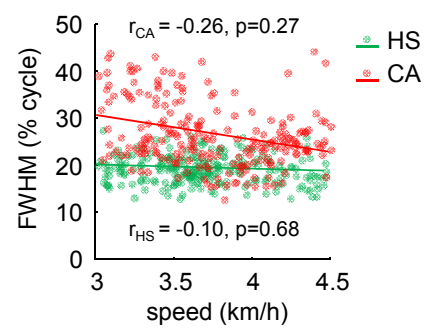

D
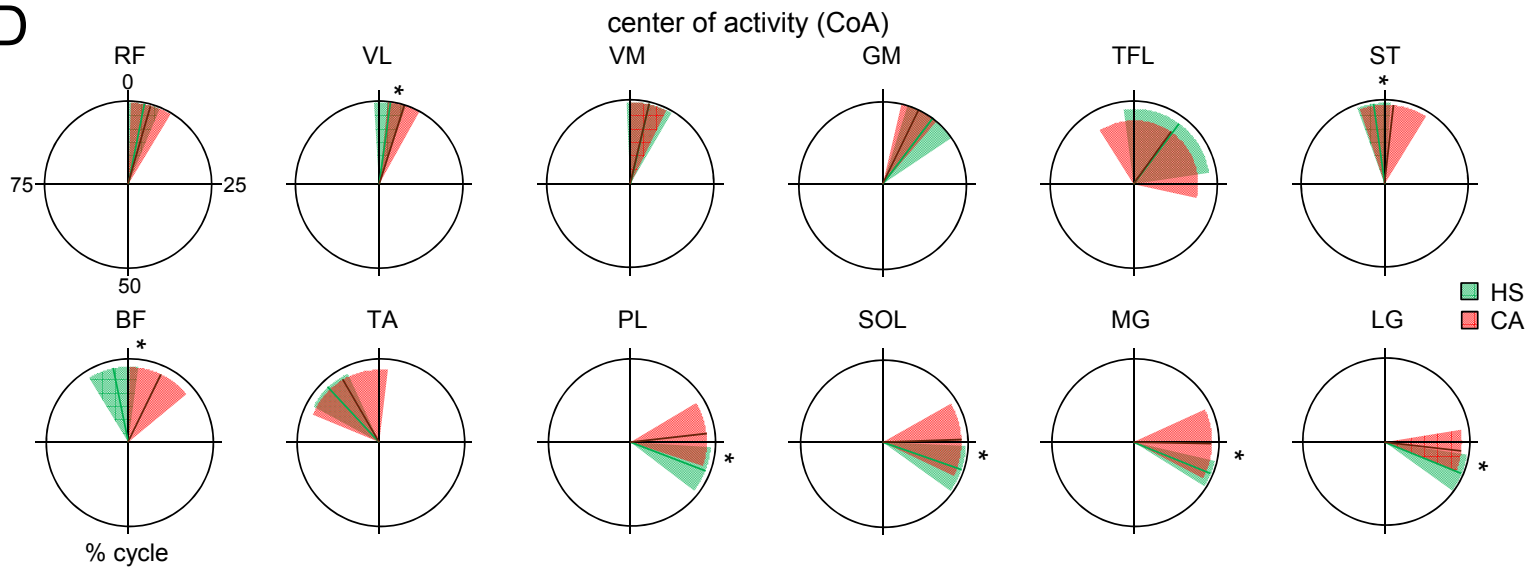

Fig. 6 


\section{A relationship between gait parameters and muscle FWHM in CA patients}
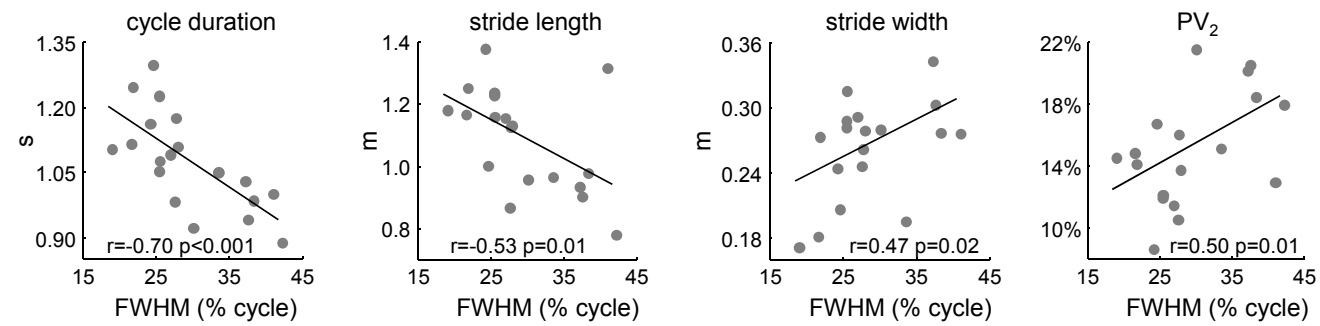

B
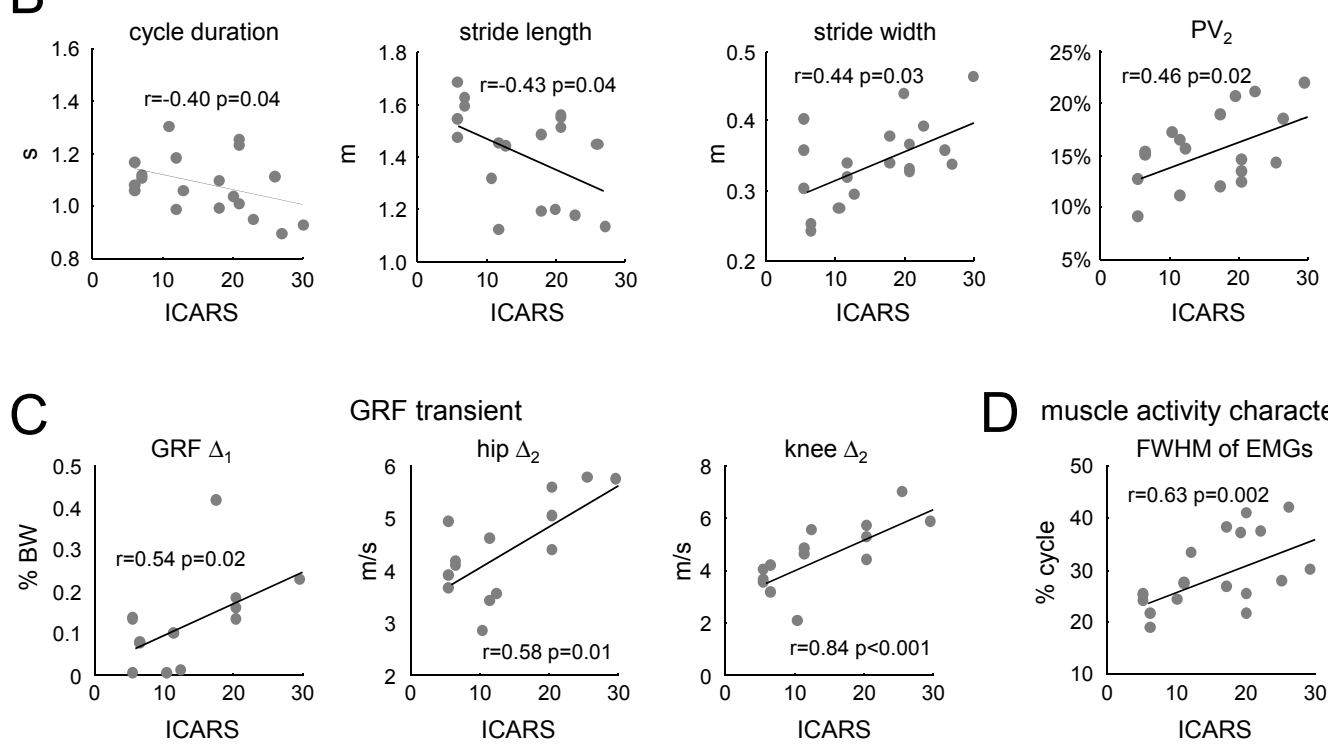

D muscle activity characteristics

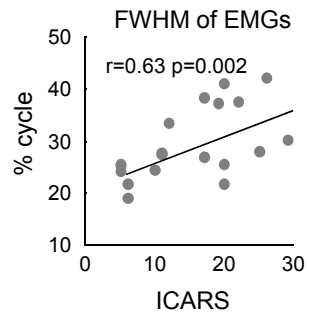

